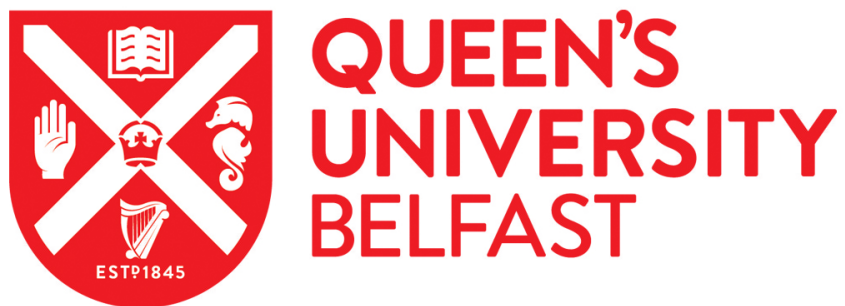

A co-operative formation-based collision avoidance approach for a group of autonomous vehicles (Special Issue on Adaptive Control and Signal Processing in Marine System)

Yang, A., Naeem, W., Fei, M., \& Tu, X. (2017). A co-operative formation-based collision avoidance approach for a group of autonomous vehicles (Special Issue on Adaptive Control and Signal Processing in Marine System). International Journal of Adaptive Control and Signal Processing, 31(4), 489-506.

https://doi.org/10.1002/acs.2563

Published in:

International Journal of Adaptive Control and Signal Processing

Document Version:

Peer reviewed version

Queen's University Belfast - Research Portal:

Link to publication record in Queen's University Belfast Research Portal

\section{Publisher rights}

Copyright (C) 2015 John Wiley \& Sons, Ltd.

This is the peer reviewed version of the following article: Yang, A., Naeem, W., Fei, M., and Tu, X. (2015), A cooperative formation-based collision avoidance approach for a group of autonomous vehicles. Int. J. Adapt. Control Signal Process, which has been published in final form at doi: 10.1002/acs.2563. This article may be used for non-commercial purposes in accordance with Wiley Terms and Conditions for Self-Archiving.

\section{General rights}

Copyright for the publications made accessible via the Queen's University Belfast Research Portal is retained by the author(s) and / or other copyright owners and it is a condition of accessing these publications that users recognise and abide by the legal requirements associated with these rights.

Take down policy

The Research Portal is Queen's institutional repository that provides access to Queen's research output. Every effort has been made to ensure that content in the Research Portal does not infringe any person's rights, or applicable UK laws. If you discover content in the Research Portal that you believe breaches copyright or violates any law, please contact openaccess@qub.ac.uk. 


\title{
A co-operative formation-based collision avoidance approach for a group of autonomous vehicles
}

\author{
Aolei Yang, Wasif Naeem, Minrui Fei, and Xiaowei Tu
}

\begin{abstract}
This paper employs a unique decentralised cooperative control method to realise a formation-based collision avoidance strategy for a group of autonomous vehicles. In this approach, the vehicles' role in the formation and their alert and danger areas are first defined, and the formation-based intra-group and external collision avoidance methods are then proposed to translate the collision avoidance problem into the formation stability problem. The extensiondecomposition-aggregation formation control method is next employed to stabilise the original and modified formations whilst manoeuvring, and subsequently solve their collision avoidance problem indirectly. Simulation study verifies the feasibility and effectiveness of the intra-group and external collision avoidance strategy. It is demonstrated that both formation control and collision avoidance problems can be simultaneously solved if the stability of the expanded formation including external obstacles can be satisfied.
\end{abstract}

\section{Index Terms}

Formation control, collision avoidance, extension-decomposition-aggregation (EDA), stability, intra-group collision, co-operative control.

\section{INTRODUCTION}

All collisions either at sea, land or in the air have serious environmental, economical, and human life implications. The sinking of the Titanic was an infamous catastrophe at sea [1] caused by collision with an iceberg. Similar unfortunate events had also occurred in the air such as the 2002 Überlingen mid-air collision between the Bashkirian Airlines Flight 2937 and the Boeing 757-23APF cargo jet in southern Germany [2]. In cooperative control applications involving multiple vehicles, this problem is further exacerbated as collision within the formation also needs to be considered. The provision of collision avoidance capability is thus considered an integral part of any manned or unmanned system. For surveillance type missions, an aircraft with such a function can safely fly at low-altitudes to minimise the possibility of detection by a radar [4], [5]. Similarly, a submarine can swim stealthily close to the seafloor to minimise the risk of detection by sonar equipment [6]. Another growing application of UAVs in civilian domain is the use of quadrotors for photography, hence safety is critical. Here, collision avoidance is explicitly defined as the capability of a vehicle (individually or within a formation) to detect and avoid collisions with unidentified objects (UO), while still attempting to accomplish the prescribed mission. In the technical sense, collision avoidance strategies are usually software-based whereas onboard sensors detect external objects/obstacles and monitor \& map the dynamic outdoor environment. Additionally, any evasive strategy should be able to provide sufficient lead time and to guide the vehicle(s) to take appropriate avoidance action [3] by making use of a decision maker which is employed to evaluate the probability of collision.

A number of approaches have been proposed to address the collision avoidance problem. These in general fall into three categories: prescribed, optimised, and reactive approaches [8]. Noting that obstacle avoidance is generally associated with path planning, in [9], an $\mathrm{A}^{*}$ algorithm was applied to find a path in the midst of obstacles and conflicts, which is a typical 'prescribed' approach. Lamiraux et al. in [10]

A. Yang, and M. Fei are with the School of Mechatronic Engineering and Automation, Shanghai University, 200072, Shanghai, P. R. China. W. Naeem is with the School of Electronics, Electrical Engineering and Computer Science, Queen's University Belfast, BT9 5AH, UK. Corresponding author: Wasif Naeem, Email: w. naeem@qub.ac.uk 
presented a hybrid method where a path planned for known obstacles is deformed to deal with unknown ones. A similar method was also proposed in [11]. For the prescribed collision avoidance approach, all vehicles would follow a set of rules which should be designed or constructed in advance. However, it is not applicable for use in unknown environments [12].

Conversely, optimised methods involve calculating the best path for all the vehicles to take cooperative actions to avoid other vehicles or obstacles. It is a look-ahead approach which operates by minimising a defined cost function [13]. Most of the control framework of this strategy is centralised, however there exists the problem of a single point of failure [14]. The reactive approach includes the likes of potential field approach [15]. In this method, the collision avoidance action is to react online to the current state of the system, rather than planning the path in advance. Attractive and repulsive forces exist between vehicles, waypoints/goal and obstacles similar to the reflexive forces between two (same or oppositely) charged particles [16], [17]. Generally speaking, this strategy is decentralised where each individual vehicle calculates its own collision avoidance action based on surrounding vehicles, goal and obstacles [18].

In addition to the above three typical categories, a number of other novel approaches can be found in the literature. For instance, job-scheduling technique [19] was employed for intersection collision avoidance (ICA) applications. Furthermore, a collision warning algorithm was proposed to apply to general traffic scenarios [20] and for intersections [21].

A robust collision avoidance approach should be able to deal with both static and dynamic obstacles present in the environment. Static obstacles include mountains, buildings, forests etc. whereas dynamic obstacles constitute other intra-group vehicles in the formation and external vehicles (friendly or hostile) that are present in the proximity of the current vehicle. Static obstacles are relatively easier to handle, and are commonly dealt with through global path planning [3]. For dynamic obstacles, the general approach is to estimate the speed and direction of motion using sensor measurements, and subsequently calculate the obstacle position over time. A simple estimator used in many applications is the Kalman filter and collision cone concept [22]. Apart from the types of obstacles, other factors such as obstacle shape and size increase the complexity of the collision avoidance problem. It is normal practice to assume that the vehicles and obstacles are surrounded by safety circles or spheres [23], which determine safe boundaries for collision avoidance purposes. In [24], such a safety circle was employed for obstacle avoidance with multiple mobile robots, where two robots cooperated within a specified area, while others waited outside it.

\section{A. Contribution of this paper}

In comparison with the above three strategies, the proposed collision avoidance approach is based on formation-based control and stability. The underlying concept is that when obstacles or UOs invade the predefined alert area of the current vehicle, the current vehicle considers these obstacles or UOs as its reaction "vehicles" in order to construct a virtual formation. Having considered all these invading vehicles/obstacles as nodes in the virtual formation topology, a formation control methodology which should be able to guarantee the stability during formation change or transformation can be employed to stabilise the augmented formation system, and simultaneously to implement the so-called formation-based collision avoidance.

This method is different from other common approaches, which are generally based on a single strategy, in that it is a hybrid method from the perspective of architecture, containing both the prescribed and reactive strategies. The prescribed one (Level 1) is used to pre-calculate the formation geometries based on the known mission map and obstacles, and the reactive one (Level 2) is designed to deal with the collision avoidance problem caused by the presence of UOs. The feasible formation control method is then employed to maintain the stability of the formation (including the UOs) during formation geometries transformation. This way, the collision avoidance problem is translated into the formation stability problem.

Specifically, the formation-based intra-group and external collision avoidances and the related algorithms are separately discussed in detail to determine the formation geometries. In simulations, the extension- 
decomposition-aggregation formation control method was employed to maintain the stability of the formation geometries and to verify the feasibility and effectiveness of the intra-group and external collision avoidances. Further, based on the formation control and stability, this method can be conveniently extended to handle the static and dynamic collision avoidance problems together.

This remainder of the paper is organised as follows. Section II presents some necessary background material and preliminaries. The formation-based collision avoidance approach is presented in Section III, which forms the bulk of the paper, including the intra-group and external collision avoidance algorithms. Simulation results are described Section IV and concluding remarks and suggestions for future work are finally provided in Section V.

\section{PRELIMINARIES}

During the formation manoeuvre, there inevitably exists the collision risk between vehicles and obstacles, and the ability of avoiding collision is also an important feature to assess the effectiveness of the formation manoeuvre, since there may be some factors that incur unexpected intra-group or vehicle collisions, for example, (1) communication between the vehicles within the formation may break temporarily or permanently due to natural or technological reasons during manoeuvring; (2) due to unknown/unexpected electromagnetic environmental disturbances, the existence of long transmission timedelay makes an impractical control input (too large amplitude), and subsequently might lead to unpredicted response; (3) the decentralised formation controller for each vehicle can be used to avoid collisions efficiently, but not necessarily guarantee collision avoidance with other vehicles. In addition, there also exists the possibility of collision with unidentified objects (UO), and it is required that the individual vehicle should have the ability of detecting obstacles or UO by using on-board sensors and activating its reflexive obstacle avoidance.

In this paper, the formation control approach is innovatively used to solve the above collision problems, which is different from general approaches based on path planning, and is called as formation-based collision avoidance. Before describing this approach, it is necessary to discuss a few related preliminaries in this section, including the roles of different vehicles in the process of collision avoidance, and the definitions of alert area and danger area for each vehicle.

\section{A. Role definition of formation vehicles}

Each vehicle within a formation is assigned a discrete variable $\mathfrak{r}_{i}$ to define its role. The set of these role variables related to a collection of $N$ vehicles is then represented as $\mathfrak{r}=\left\{\mathfrak{r}_{i}: i=1,2, \cdots, N\right\}$, the value of which depends on the current formation control problem under consideration. It is assumed that the role of a vehicle can be changed at any time, that can be written as (1) [25],

$$
\mathfrak{r}_{i}^{\prime}=r\left(\mathbf{x}_{i}, \mathfrak{r}_{i}\right)
$$

where $\mathfrak{r}_{i}^{\prime}$ is the new value of the role variable $\mathfrak{r}_{i}$ which can be written as $\mathfrak{r}_{i}(t)$ defining the role of the $i^{t h}$ vehicle at time $t$.

Specifically, two kinds of definitions are presented here to facilitate the following discussion of the collision avoidance approach. The first one is the so-called "reference vehicle (RV)" [26] defined in a formation, and the other is non-RV (NRV). The RV is chosen by consensus between vehicles, to be the reference point of the overall formation shape. Its main tasks are outlined as follows:

1) The RV should have the capability of communicating with the remote base station, maintaining and updating the current tasks according to operator's commands.

2) The RV should have the ability of formation management and maintenance, i.e. updating the formation topology (FT) and achieving the future formation geometry (FG), based on the external changing environment.

Here, item 1 above is assumed to be satisfied, and item $\mathbf{2}$ is the focus as it is closely related to the topic of the formation-based collision avoidance. 
When changing the formation topology, two modes are defined: positive accept mode and active discard mode. The former means that there are many requests from external vehicles to apply for joining the current formation system, or an internal vehicle may apply for leave the current formation system. The latter means that if one vehicle moves away beyond the maximal radio communication range or there is communication failure for the vehicle in the formation, this vehicle should be deserted by the current formation system and the remaining vehicles will rearrange themselves as well as generating a new formation topology. Note that the second mode is referred to as the desertion problem in this paper, and vehicle breakdown can be considered as a case of the desertion problem. In addition, the capability of calculating the future formation geometry can be used to support the implementation of collision avoidance. For example, based on an area map and positions of obstacles which are known a priori, the RV can calculate a sequence of formation geometries to guarantee collision avoidance for the whole group.

The task of NRVs is relatively simple, which is to maintain the formation stability with respect to reference formation commands and changing environment. However, each NRV should have the capability of taking over RV's role, in order to continue the formation manoeuvring mission in case of RV breakdown. This will improve the robustness of formation manoeuvre under uncertain environments, provide the multivehicle formation having the capability of rebuilding and self-repairing the formation structure, and finally increase mission success rate.

Compared to the above definitions of vehicle's roles (RV and NRV), the second role definition is based on the formation topological structure. An illustration of the vehicle's role definition is shown in Fig. 1, where the whole formation topology can be expressed as (2).

$$
\mathbf{E}=\left\{\mathrm{T}_{2 \rightarrow 1}^{\mathrm{F}}, \mathrm{T}_{1 \leftrightarrow 3}^{\mathrm{F}}, \mathrm{T}_{2 \leftrightarrow 3}^{\mathrm{F}}, \mathrm{T}_{2 \leftrightarrow 4}^{\mathrm{F}}, \mathrm{T}_{4 \rightarrow 3}^{\mathrm{F}}, \mathrm{T}_{5 \rightarrow 4}^{\mathrm{F}}, \mathrm{T}_{5 \rightarrow 3}^{\mathrm{F}}\right\}
$$

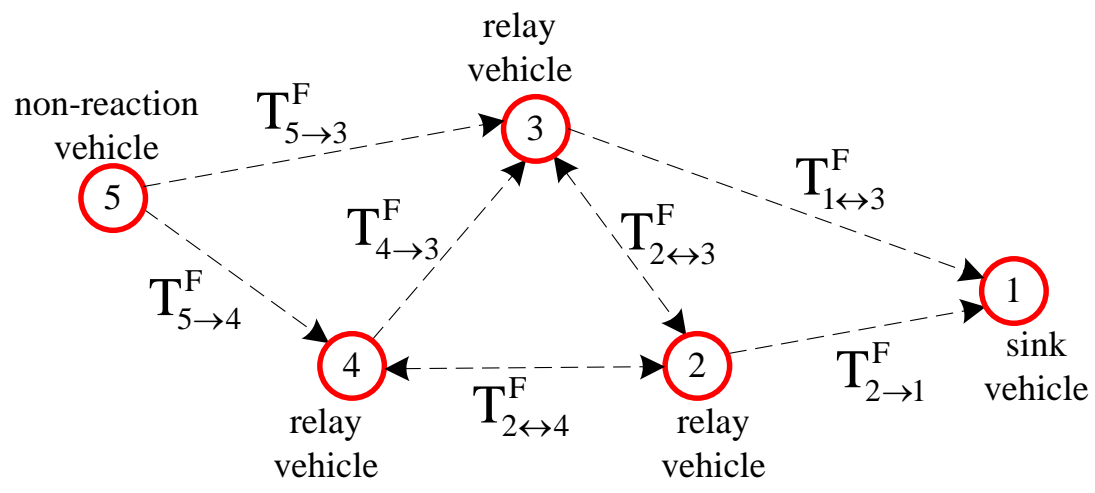

Fig. 1. Role definition based on the formation topology

Three kinds of roles: sink, relay and non-reaction vehicles are proposed here to describe each vehicle's function in the formation topology. In Fig. 1, the sink vehicle (vehicle 1) is defined to represent the vehicle which only receives data from other vehicles or which only reacts to other vehicles actions. The relay vehicle has a "relay" function, which both transmits and receives data from its interconnecting vehicles, such as vehicle 2, 3 and 4 . The last type is the non-reaction vehicle which only transmits data, and it does not react to any other vehicles in the formation, such as vehicle 5 in Fig. 1. In general, the relay vehicles are vital for a robust formation, whereas the other two roles rarely appear unless for a special purpose. For example, a dispensable vehicle is commonly designated as the sink vehicle, and a UO is always considered as a non-reaction vehicle to make the UO become a part of the formation.

The two definitions above are generally not independent, and important considerations are required as follows:

1) The sink vehicle cannot be the RV in the formation.

2) In order to improve the response speed, the relay vehicle with maximal interconnections to other vehicles is generally selected as the RV. 
3) As for the leader-following formation control strategy, the leader of the overall formation is usually the non-reaction vehicle.

\section{B. Alert area and overlap area}

As shown in Fig. 2, zones are defined around the $i^{\text {th }}$ vehicle, where the inner area surrounded by the

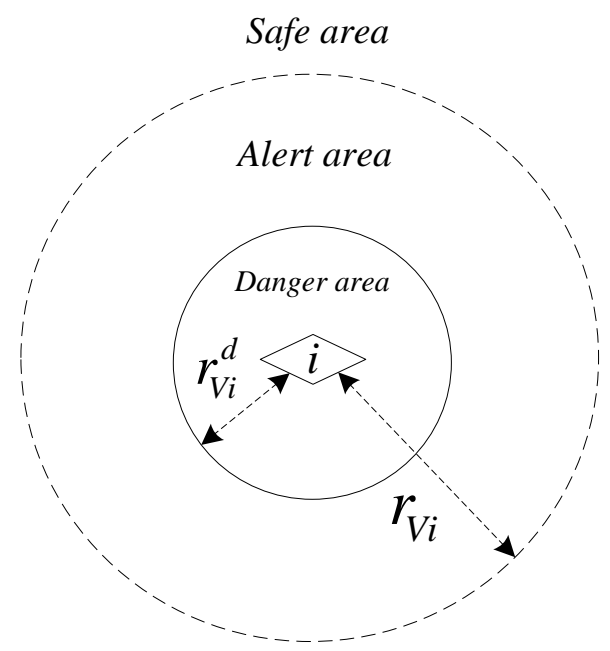

Fig. 2. Definition of safe, alert and danger areas for the $i^{\text {th }}$ vehicle

solid circle is called the danger area, the circle's radius is $r_{V i}^{d}$ and its superscript " $d$ " denotes danger. The annular area between the inner circle and the outer dotted circle with radius $r_{V i}$ is termed as the alert area, whilst the area outside the alert area is designated as the safe area. During normal operation, other objects should stay in the safe area of the $i^{\text {th }}$ vehicle. When an object enters into the alert area due to an unexpected reason, the vehicle should activate its collision avoidance routine in order to move itself away from the invading object. Any objects should not be permitted to enter into the danger area because of high collision possibility.

Also, within a formation, vehicles located at different positions might have different alert radii, so there is a possibility of the alert area overlapping. As shown in Fig. 3, the alert areas of the $i^{t h}, j^{t h}$ and

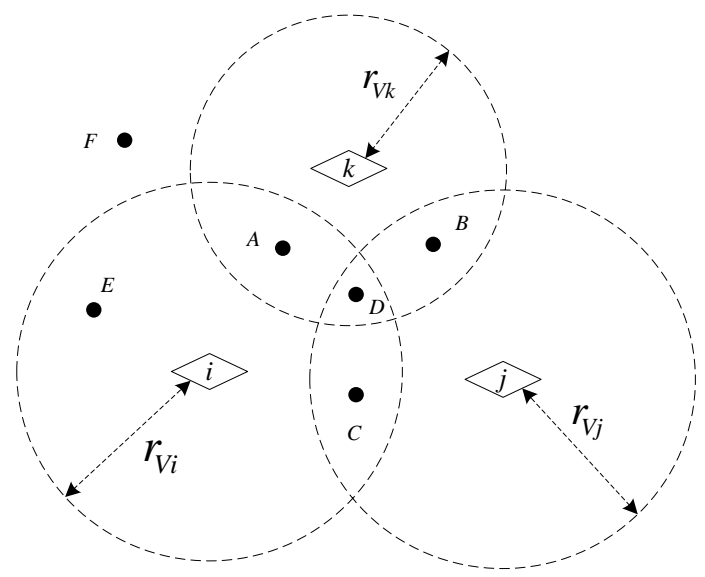

Fig. 3. An illustration of overlapped area for the $i^{t h}, j^{t h}$ and $k^{\text {th }}$ vehicles

$k^{\text {th }}$ vehicles overlap each another. Specifically, points $A, B$ and $C$ lie in the overlap areas related to two vehicles' alert areas, and these corresponding areas are then called as 2-fold overlap areas. By the same 
token, point $D$ can be considered as a point in the 3-fold overlap area, and points $E$ and $F$ are in 1 -fold and 0-fold overlap areas, respectively.

\section{FORMATION-BASED COLLISION AVOIDANCE}

\section{A. Formation-based collision avoidance methodology}

The proposed formation-based collision avoidance can be described briefly as follows: when obstacles or UOs invade into the predefined alert area of the current vehicle, it considers these obstacles or UOs as its reaction vehicles. In other words, these obstacles will be considered as a part of the whole "virtual" formation topology once there appears the possibility of a collision. Collision avoidance can then be implemented by tracking a sequence of feasible formation geometries designed to avoid those UOs.

In order to implement this idea, two requirements are to be satisfied:

1) A sequence of feasible formation geometries related to known or unknown maps and obstacles should be calculated by using prescribed and reactive strategies;

2) The employed formation control methodology should be able to guarantee the stability of the constructed virtual formation during any cooperative transformation.

Here, the formation-based collision avoidance strategy contains two stages: deliberative pre-computation of collision avoidance and reflexive or reactive collision avoidance strategy, which is shown in Fig. 4. The

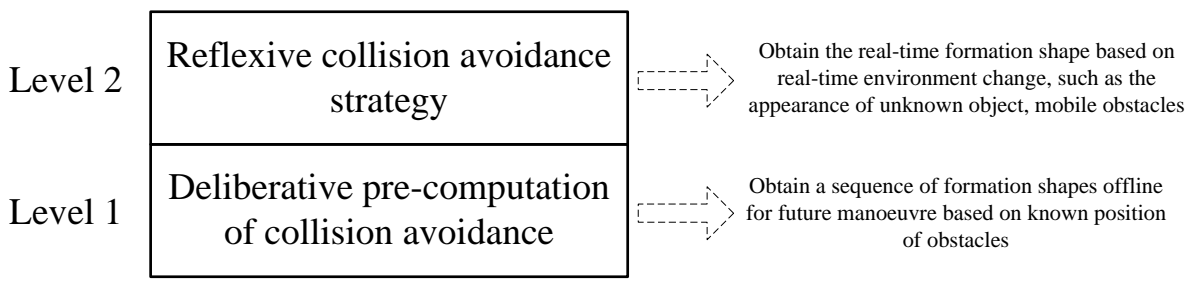

Fig. 4. Formation-based collision avoidance strategy

first level is an offline approach, where a sequence of formation geometries or formation shapes can be first pre-calculated under the assumption that the mission map and position of static obstacles are known a priori. These achieved formation shapes are then in turn selected as the real-time reference formations at specific locations. It is noted that Level 1 is different from simple path planing, and the procedure of designing a formation shape is briefly listed below:

1) Calculate waypoints from start point to destination and construct a desired path.

2) Based on the positions and sizes of all known obstacles, successively search the achieved path and determine the relationship between the lateral width $\left(w_{F}\right)$ of the current formation shape and the maximum clearance $\left(w_{C}\right)$ between these obstacles.

3) If $\mathrm{w}_{F}<\mathrm{w}_{C}$, it is not necessary to change the current formation geometry, whereas if $\mathrm{w}_{F} \geq \mathrm{w}_{C}, \mathrm{a}$ new formation shape is needed to be calculated, as well as determining a desired position where formation will be changed.

4) For simplicity, the adopted strategy for designing a desired formation shape is to give priority to regulating the lateral formation, and to consider the change of the longitudinal formation as a secondary measure which is employed only when the primary measure is not sufficient to guarantee a collision-free path.

In contrast to the deliberative pre-computation of a collision avoidance manoeuvre in Level 1, Level 2 is a reflexive strategy which is designed to enhance the robustness to the varying environment and to deal with emergent obstacles. Various functions in Level 2 are given as follows:

1) Avoid intra-group collision, whether a vehicle within a formation is uncontrollable due to its hardware/software breakdown, or a vehicle receiving incorrect/incomplete commands poses a risk to other vehicles in the formation. 
2) Avoid any external obstacles as there always exists information discrepancy between the known map status and the real-time environment.

3) Avoid stationary and mobile/dynamic UOs. Particularly, the mobile obstacles avoidance approach has potentially wide application prospects.

The central role of Level 2 is to reconstruct and update the formation structure online as well as to change the formation geometry based on real-time knowledge about the external environment. Its tasks are detailed as follows:

1) Once a local vehicle is confirmed as malfunctioned, discard the vehicle from the original formation or delete the corresponding node in the formation topology.

2) Once an UO invades into the predefined alert area of the $i^{t h}$ vehicle, the related vehicle will accept the UO as its reaction target. This means that the original formation system is augmented with the UO and a new formation topology is created. This newly generated formation is termed as an augmented formation.

3) Determine a sequence of new formation geometries in real time to make the original formation move away from the newly joined UO as quickly as possible.

4) Change and update the current formation topology to optimise the formation reaction structure, and consequently improve the dynamic performance during formation change and obstacle avoidance.

The implementation of task 1 above depends on consensuses among the remaining vehicles and the pre-designed formation self-construction protocol. If the formation connectivity remains undisturbed after removing the problem vehicle, the corresponding node can be directly deleted from the original topology. Otherwise, the original formation topology has to be reconstructed based on a relevant protocol. Research on these self-construction protocols is another broad field, which is not the focus of this paper. In addition, task 4 above is related to finding criteria about how to determine a formation topology which has a better formation change performance than others. It is deemed important to devote a separate paper to discuss which of the selected formation topologies is better than others by carrying out comparative simulations.

The proposed approach for task 2 and 3 will be discussed in the following for two cases: intra-group collision avoidance and external collision avoidance.

\section{B. Intra-group collision avoidance}

The schematic diagram shown in Fig. 5 illustrates the method of determining an appropriate formation geometry and for modifying the current formation topology for avoiding intra-group collision. This scenario is referred to as internal collision avoidance which mostly occurs when an inner vehicle invades into the alert area of neighbouring vehicles due to unexpected external disturbances, communication errors or malfunctioning. It is assumed that each vehicle in the formation is able to detect any invading objects by using on-board sensors. Once an object has been detected by the $i^{t h}$ vehicle within its alert area, it immediately activates its collision avoidance routine. The ultimate aim is to keep the invading vehicle at the minimum safe distance marked by the boundary of the alert area in Fig. 2. Note that the danger circle of each vehicle is not shown in Fig. 5 to avoid any confusion.

In Fig. 5 (A), the formation representation ( $F R$, including formation geometry and topology) for a group of 4 vehicles using bidirectional communications is illustrated, where the diamond symbolises a vehicle, and the dotted circles with radii $r_{V 1}, r_{V 2}$ and $r_{V 3}$ indicate the alert areas for the $1^{\text {st }}, 2^{\text {nd }}$ and $3^{\text {rd }}$ vehicles respectively. In this case, the $4^{\text {th }}$ vehicle is assumed to be moving into the alert area of the $1^{s t}$ vehicle, where $\mathbf{p}_{L 4}^{r}$ denotes the real or actual position in 1-fold overlap area. It must be underlined that the small circle labelled by $\mathbf{p}_{L 4}^{d}$ is the point joining the alert circle and the extended line of the vector $\overrightarrow{\mathbf{p}_{L 1} \mathbf{p}_{L 4}^{r}}$, which is also the nearest point from the alert circle to the current position of the $4^{\text {th }}$ vehicle.

The proposed solution is to change the reference formation from the original $F R_{r e f}$ to a newly designed or desired $F R_{\text {real }}^{d}$. The related formation representations are listed in Table I. 


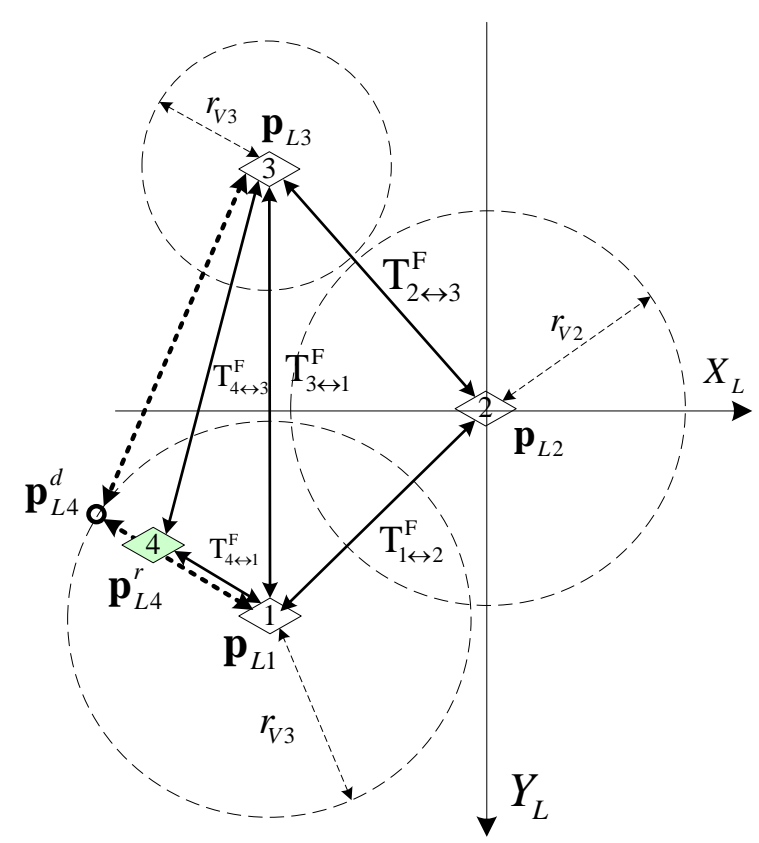

(A)

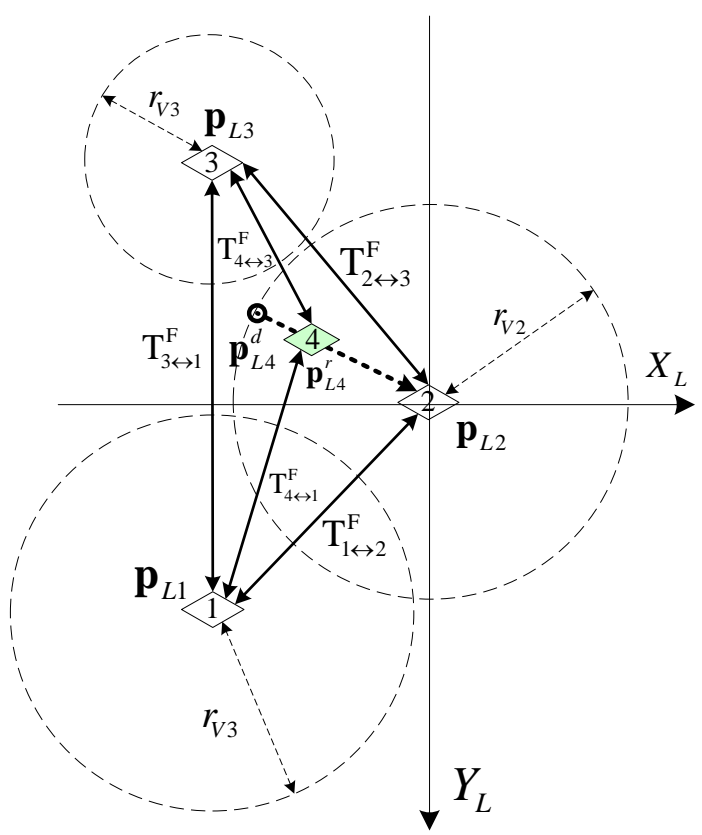

(B)

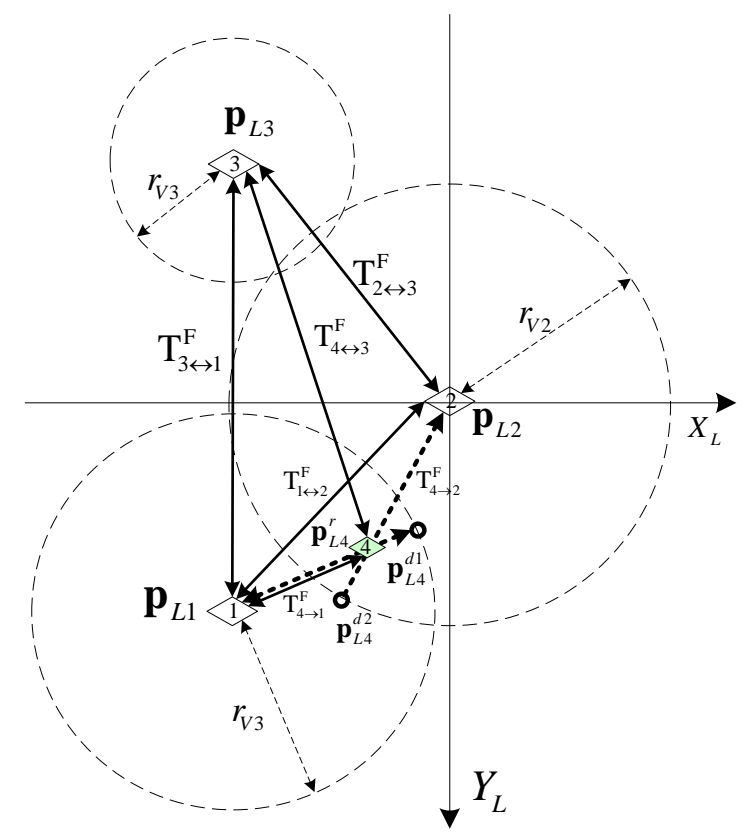

(C)

Fig. 5. Intra-group vehicles collision avoidance

Note that there are no changes to the topology, since there already exists a direct formation reaction defined by $\mathrm{T}_{4 \leftrightarrow 1}^{\mathrm{F}}$. However, the formation geometry has to be changed from $\left\{\mathbf{p}_{L 1}, \mathbf{p}_{L 2}, \mathbf{p}_{L 3}, \mathbf{p}_{L 4}\right\}$ to $\left\{\mathbf{p}_{L 1}, \mathbf{p}_{L 2}, \mathbf{p}_{L 3}, \mathbf{p}_{L 4}^{d}\right\}$.

On the other hand, as shown in Fig. 5 (B), the $4^{\text {th }}$ vehicle invades the alert area (1-fold overlap area) of the $2^{\text {nd }}$ vehicle which has no direct reaction to the invading vehicle. Owing to the non-existence of reaction between the $2^{\text {nd }}$ vehicle and $4^{\text {th }}$ vehicle, the original formation topology needs to be augmented with $\mathrm{T}_{4 \rightarrow 2}^{\mathrm{F}}$, where $\mathbf{p}_{L 4}^{d}$ is the desired reference position for the $4^{t h}$ vehicle. The related sequence showing 
TABLE I

SEQUENCE OF FORMATION REPRESENTATION CORRESPONDING TO FIG. 5 (A)

\begin{tabular}{c|c|c}
\multirow{2}{*}{ Sequence } & \multicolumn{2}{|c}{ Formation representation } \\
\cline { 2 - 3 } Current $F R_{\text {real }}$ & Formation geometry & Formation topology \\
Original $F R_{r e f}$ & $\left\{\mathbf{p}_{L 1}, \mathbf{p}_{L 2}, \mathbf{p}_{L 3}, \mathbf{p}_{L 4}^{r}\right\}$ & $\left\{\mathrm{T}_{1 \leftrightarrow 2}^{\mathrm{F}}, \mathrm{T}_{2 \leftrightarrow 3}^{\mathrm{F}}, \mathrm{T}_{3 \leftrightarrow 4}^{\mathrm{F}}, \mathrm{T}_{4 \leftrightarrow 1}^{\mathrm{F}}, \mathrm{T}_{3 \leftrightarrow 1}^{\mathrm{F}}\right\}$ \\
New desired $F R_{r e f}^{d}$ & $\left\{\mathbf{p}_{L 1}, \mathbf{p}_{L 2}, \mathbf{p}_{L 3}, \mathbf{p}_{L 4}\right\}$ & $\left\{\mathrm{T}_{1 \leftrightarrow 2}^{\mathrm{F}}, \mathrm{T}_{2 \leftrightarrow 3}^{\mathrm{F}}, \mathrm{T}_{3 \leftrightarrow 4}^{\mathrm{F}}, \mathrm{T}_{4 \leftrightarrow 1}^{\mathrm{F}}, \mathrm{T}_{3 \leftrightarrow 1}^{\mathrm{F}}\right\}$ \\
$\left\{\mathbf{p}_{L 1}, \mathbf{p}_{L 2}, \mathbf{p}_{L 3}, \mathbf{p}_{L 4}^{d}\right\}$ & $\left\{\mathrm{T}_{1 \leftrightarrow 2}^{\mathrm{F}}, \mathrm{T}_{2 \leftrightarrow 3}^{\mathrm{F}}, \mathrm{T}_{3 \leftrightarrow 4}^{\mathrm{F}}, \mathrm{T}_{4 \leftrightarrow 1}^{\mathrm{F}}, \mathrm{T}_{3 \leftrightarrow 1}^{\mathrm{F}}\right\}$
\end{tabular}

the representations are given in Table II.

TABLE II

SEQUENCE OF FORMATION REPRESENTATION CORRESPONDING TO FIG. 5 (B)

\begin{tabular}{c|c|c}
\multirow{2}{*}{ Sequence } & \multicolumn{2}{|c}{ Formation representation } \\
\cline { 2 - 3 } Current $F R_{\text {real }}$ & Formation geometry & Formation topology \\
Original FR ref & $\left\{\mathbf{p}_{L 1}, \mathbf{p}_{L 2}, \mathbf{p}_{L 3}, \mathbf{p}_{L 4}^{r}\right\}$ & $\left\{\mathrm{T}_{1 \leftrightarrow 2}^{\mathrm{F}}, \mathrm{T}_{2 \leftrightarrow 3}^{\mathrm{F}}, \mathrm{T}_{3 \leftrightarrow 4}^{\mathrm{F}}, \mathrm{T}_{4 \leftrightarrow 1}^{\mathrm{F}}, \mathrm{T}_{3 \leftrightarrow 1}^{\mathrm{F}}\right\}$ \\
New desired $F R_{\text {ref }}^{d}$ & $\left\{\mathbf{p}_{L 1}, \mathbf{p}_{L 2}, \mathbf{p}_{L 3}, \mathbf{p}_{L 4}\right\}$ & $\left\{\mathrm{T}_{1 \leftrightarrow 2}^{\mathrm{F}}, \mathrm{T}_{2 \leftrightarrow 3}^{\mathrm{F}}, \mathrm{T}_{3 \leftrightarrow 4}^{\mathrm{F}}, \mathrm{T}_{4 \leftrightarrow 1}^{\mathrm{F}}, \mathrm{T}_{3 \leftrightarrow 1}^{\mathrm{F}}\right\}$ \\
$\left\{\mathbf{p}_{L 1}, \mathbf{p}_{L 2}, \mathbf{p}_{L 3}, \mathbf{p}_{L 4}^{d}\right\}$ & $\left\{\mathrm{T}_{1 \leftrightarrow 2}^{\mathrm{F}}, \mathrm{T}_{2 \leftrightarrow 3}^{\mathrm{F}}, \mathrm{T}_{3 \leftrightarrow 4}^{\mathrm{F}}, \mathrm{T}_{4 \leftrightarrow 1}^{\mathrm{F}}, \mathrm{T}_{3 \leftrightarrow 1}^{\mathrm{F}}, \mathrm{T}_{4 \rightarrow 2}^{\mathrm{F}}\right\}$
\end{tabular}

For the third case depicted in Fig. $5(\mathrm{C})$, the $4^{\text {th }}$ vehicle enters into the 2-fold overlap alert area of the $1^{\text {st }}$ and $2^{\text {nd }}$ vehicles. This scenario can be considered as the resultant of the two cases shown in Fig. 5 (A) and Fig. 5 (B). The previously proposed approach is also suitable to deal with the collision avoidance problem in this overlapped area. However, it is noted that there exist two candidates ( $\mathbf{p}_{L 4}^{d 1}$ and $\mathbf{p}_{L 4}^{d 2}$ ) of the desired reference position for the $4^{t h}$ vehicle. A feasible solution to this problem is to calculate the arithmetic mean of $\mathbf{p}_{L 4}^{d 1}$ and $\mathbf{p}_{L 4}^{d 2}$ as the desired reference position. The relevant sequence of formation representation is thus listed in Table III.

TABLE III

SEQUENCE OF FORMATION REPRESENTATION CORRESPONDING TO FIG. 5 (C)

\begin{tabular}{c|l|l}
\multirow{2}{*}{ Sequence } & \multicolumn{2}{|c}{ Formation representation } \\
\cline { 2 - 3 } Current $F R_{\text {real }}$ & Formation geometry & Formation topology \\
Original $F R_{\text {ref }}$ & $\left\{\mathbf{p}_{L 1}, \mathbf{p}_{L 2}, \mathbf{p}_{L 3}, \mathbf{p}_{L 4}^{r}\right\}$ & $\left\{\mathrm{T}_{1 \leftrightarrow 2}^{\mathrm{F}}, \mathrm{T}_{2 \leftrightarrow 3}^{\mathrm{F}}, \mathrm{T}_{3 \leftrightarrow 4}^{\mathrm{F}}, \mathrm{T}_{4 \leftrightarrow 1}^{\mathrm{F}}, \mathrm{T}_{3 \leftrightarrow 1}^{\mathrm{F}}\right\}$ \\
New desired $F R_{\text {ref }}^{d}$ & $\left\{\mathbf{p}_{L 1}, \mathbf{p}_{L 2}, \mathbf{p}_{L 3}, \frac{\left.\mathbf{p}_{L 4}{ }_{L 4}\right\}}{2}, \mathbf{p}_{L 4}^{d 2}\right\}$ & $\left\{\mathrm{T}_{1 \leftrightarrow 2}^{\mathrm{F}}, \mathrm{T}_{2 \leftrightarrow 3}^{\mathrm{F}}, \mathrm{T}_{3 \leftrightarrow 4}^{\mathrm{F}}, \mathrm{T}_{4 \leftrightarrow 1}^{\mathrm{F}}, \mathrm{T}_{3 \leftrightarrow 1}^{\mathrm{F}}\right\}$ \\
$\left\{\mathrm{T}_{1 \leftrightarrow 2}^{\mathrm{F}}, \mathrm{T}_{2 \leftrightarrow 3}^{\mathrm{F}}, \mathrm{T}_{3 \leftrightarrow 4}^{\mathrm{F}}, \mathrm{T}_{4 \leftrightarrow 1}^{\mathrm{F}}, \mathrm{T}_{3 \leftrightarrow 1}^{\mathrm{F}}, \mathrm{T}_{4 \rightarrow 2}^{\mathrm{F}}\right\}$
\end{tabular}

Finally, once the invading vehicle has moved to the safe area with regards to the formation, the interrupt routine (emergent task) of the collision avoidance is completed, and the formation system will restore to its previous task before the disruption.

The above discussion to the intra-group collision avoidance approach is based on the perspective of the global formation representation. Although it is convenient to analyse, it cannot be directly applied to the decentralised control framework. It is therefore necessary to decompose the previous global formation expression into a group of local forms or representations, which will be demonstrated in the following subsection.

In a decentralised control framework, each vehicle can react or communicate with some of other vehicles in the formation based on the predefined formation topology. When the $j^{\text {th }}$ vehicle invades the alert area of the $i^{t h}$ vehicle, the collision avoidance strategy can be expressed as Algorithm 1, which is the routine executed by the $i^{t h}$ vehicle during its collision avoidance stage.

It is noted that when the $j^{t h}$ vehicle is in a $N$-fold overlap alert area, this decentralised algorithm remains suitable for all the vehicles. In this scenario, each vehicle, including the invading vehicle, will make use of Algorithm 1 to avoid collision with other vehicles, and consequently their joint efforts 
Input: Alert area radius $r_{V i}$ of the $i^{t h}$ vehicle, current reference $L F R_{r_{e} f_{-}}$, current real $L F R_{\text {real_}} i$. Note that $L F R$ contains of the related local formation geometry $(L F G)$ and formation topology $(L F T)$.

Output: Next step reference local formation representation $L F R_{\text {ref_i }} i$

1 Calculate the current position of the $i^{t h}$ vehicle $\mathbf{p}_{L i}$ based on $L F R_{\text {real_i }}$;

2 Calculate the current position of the $j^{\text {th }}$ vehicle $\mathbf{p}_{L j}^{r}$ based on $L F R_{\text {real }} i$;

3 Calculate the reference position of the $j^{t h}$ vehicle $\mathbf{p}_{L j}^{d}$ based on $L F R_{\text {ref }} i$;

4 Determine the relative distance: $\Delta d_{\text {real }}=\left\|\mathbf{p}_{L i} \mathbf{p}_{L j}^{r}\right\|$;

5 if $\Delta d_{\text {real }} \leq r_{V i}$ then

6 Achieve the crossing point coordinate $\mathbf{p}_{\text {cross }}$ between the alert area circle and the extended line of the vector $\overrightarrow{\mathbf{p}_{L i} \mathbf{p}_{L j}^{r}}$;

$7 \quad$ Based on $L F R_{\text {real_i }}$, determine whether there exists a direct reaction to the $j^{\text {th }}$ vehicle;

if existence then

Update the desired position of the $j^{t h}$ vehicle: $\mathbf{p}_{L j}^{d}=\mathbf{p}_{\text {cross }}$;

Update the reference local formation geometry: $L F G_{r e f_{-} i}$;

Update the reference local formation representation: $L F R_{r e f_{-} i}=L F R_{r e f_{-} i}^{d}$; else

Add a reaction topology of the $i^{\text {th }}$ vehicle relative to the $j^{\text {th }}$ vehicle;

Update the reference local formation topology: $L F T_{\text {ref_i }} i$;

Update the desired position of the $j^{t h}$ vehicle: $\mathbf{p}_{L j}^{d}=\mathbf{p}_{\text {cross }}$;

Update the reference local formation geometry: $L F G_{r e f_{-} i}$;

Update the reference local formation representation: $L F R_{r e f_{-} i}=L F R_{r e f_{-} i}^{d}$;

end

return the updated $L F R_{r e f_{-} i}$;

20 else

21 return original reference local formation representation: $L F R_{r e f_{-} i}$;

2 end

Algorithm 1: Algorithm of intra-group collision avoidance for the $i^{\text {th }}$ vehicle

result in the realisation of the global collision avoidance. Besides the intra-group collision avoidance, the collision problem between a multi-vehicle formation and external UOs will be dealt with in the following subsection.

\section{Formation collision avoidance for external obstacles}

The formation collision avoidance for external obstacles has more complex impact factors in uncertain environment. In contrast to the intra-group collision avoidance, the collision avoidance problem here will be discussed from the perspective of the decentralised framework. The local or individual formation representation for each vehicle is adopted to facilitate the presentation of the proposed strategy.

As shown in Fig. 6, the object labelled "4" denotes an UO, whose velocity is unknown to the other vehicles in the formation, however its position can be determined by each vehicle. It can also be observed that Fig. 6 (A) (D) illustrates a complete passing process of the UO. Specifically, Figs. 6 (A) and (C) depict the UO in the alert area of the $2^{\text {nd }}$ and $1^{\text {st }}$ vehicles, respectively. Fig. 6 (B) shows that the UO enters into a 2 -fold overlap alert area between the $1^{\text {st }}$ and $2^{\text {nd }}$ vehicles. Fig. 6 (D) indicates that the UO has left all the alert areas.

Since there exists no topological connectivity between the formation vehicles and UOs, it is necessary to augment the original formation topology. A typical illustration being shown in Fig. 6 is presented to 


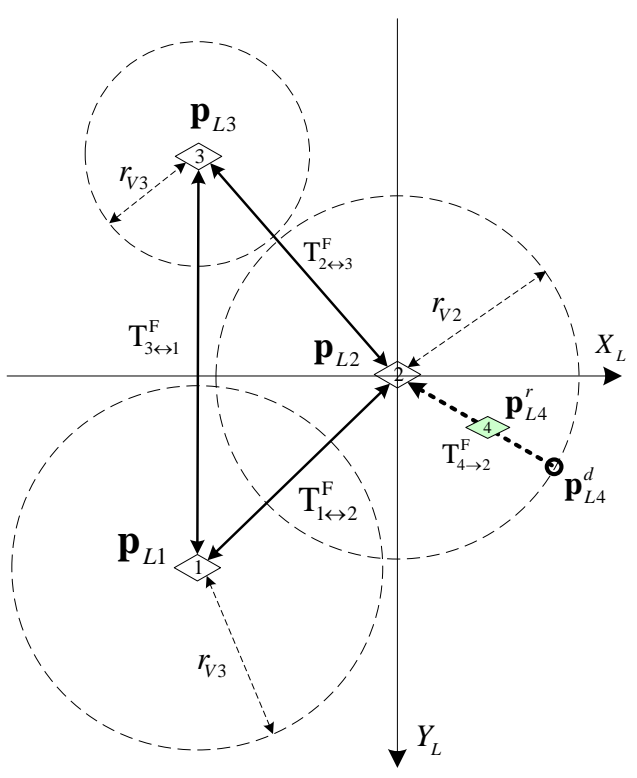

(A)

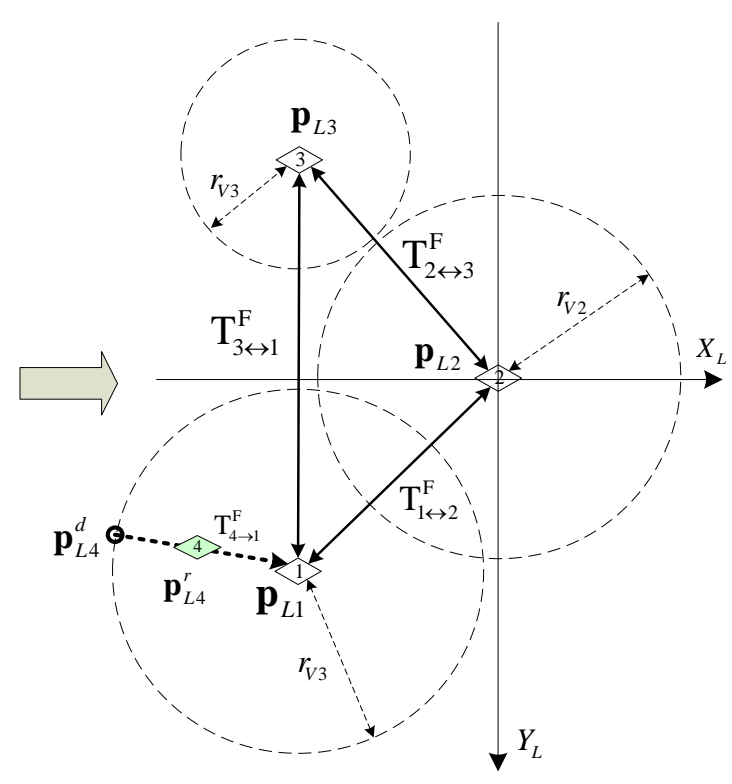

(C)

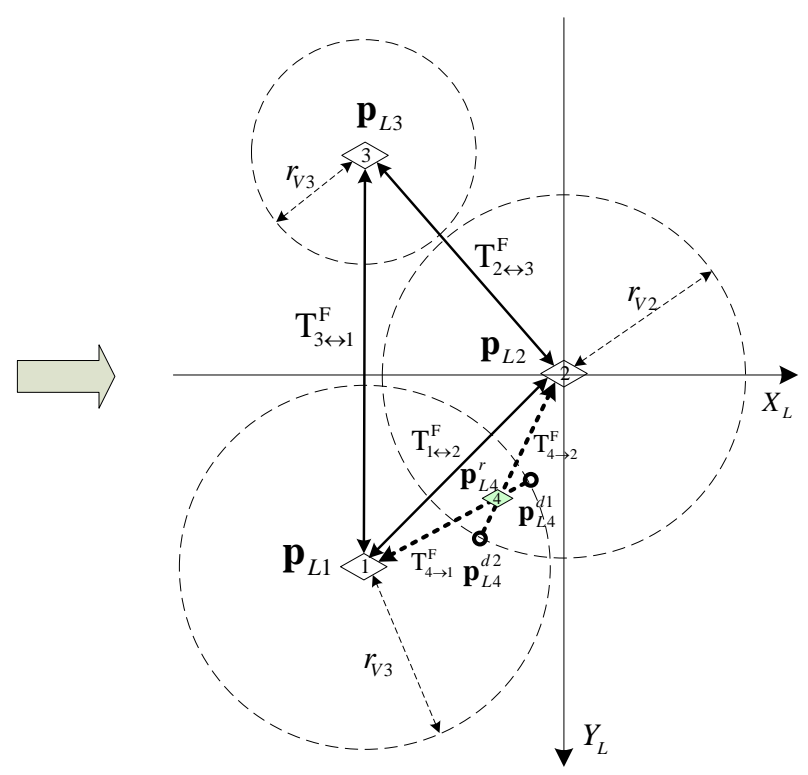

(B)

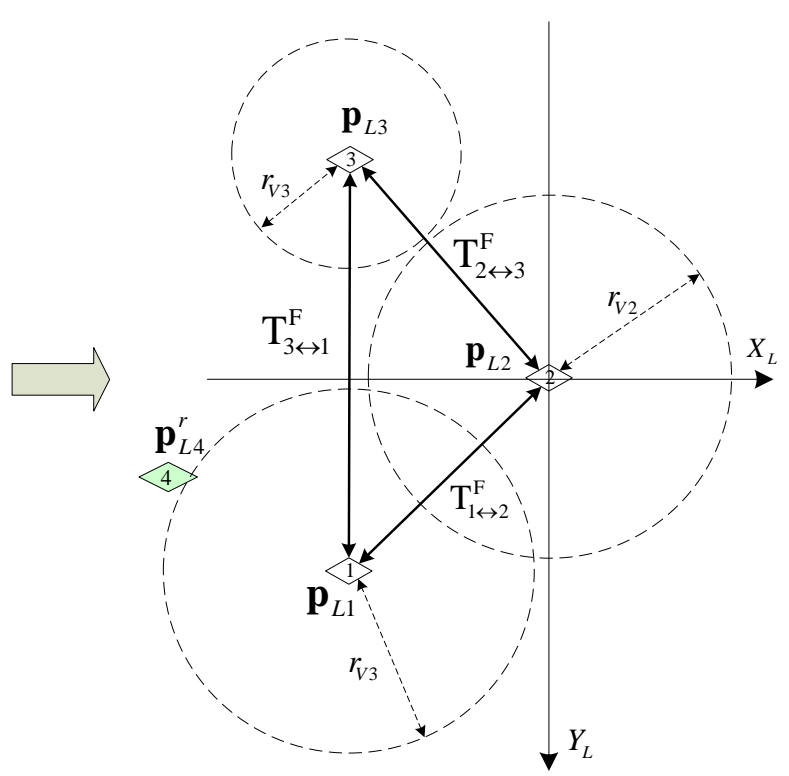

(D)

Fig. 6. Formation external collision avoidance

explain the strategy. When the UO is in the alert area of the $2^{\text {nd }}$ vehicle as shown in Fig. 6 (A), the $2^{\text {nd }}$ vehicle needs to augment its original formation topology with an unidirectional topology branch $\mathrm{T}_{4 \rightarrow 2}^{\mathrm{F}}$, as well as to change its reference local formation geometry. On the other hand, when the UO invades the 2 -fold overlap alert area as shown in Fig. 6 (B), the $1^{\text {st }}$ and $2^{\text {nd }}$ vehicles will individually consider the UO as their invader. In other words, each vehicle in the topology is unnecessary to care about whether or not other vehicles have already considered the UO as their invader. This strategy for avoiding external collisions within the 1-fold alert area is also applicable to solve the collision avoidance problem occurring in an $\mathrm{N}$-fold alert area. In Fig. 6, the process of the UO passing through the formation results in a new desired local formation representation, $L F R_{r e f_{-}}^{d}$, where the local formation of each vehicle is listed in Table IV. 
TABLE IV

NEW DESIRED LOCAL FORMATION REPRESENTATION DURING COLLISION AVOIDANCE CORRESPONDING TO FIG. 6 (A) $\sim(D)$, WHERE $L F R_{r e f i}^{d}(i=1,2,3)$ DENOTES THE DESIRED LOCAL FORMATION REPRESENTATION OF THE $i^{\text {th }}$ VEHICLE, AND $L F G_{i}, L F T_{i}$ CORRESPOND TO THE LOCAL FORMATION GEOMETRY AND THE LOCAL FORMATION TOPOLOGY, RESPECTIVELY.

\begin{tabular}{|c|c|c|c|}
\hline \multicolumn{2}{|c|}{ Formation vehicles } & Fig. 6 (A) & Fig. 6 (B) \\
\hline Vehicle 1: $L F R_{r e f_{-} 1}^{d}$ & $\begin{array}{l}L F G_{1} \\
L F T_{1}\end{array}$ & $\begin{array}{l}\left\{\mathbf{p}_{L 1}, \mathbf{p}_{L 2}, \mathbf{p}_{L 3}\right\} \\
\left\{\mathrm{T}_{1 \leftrightarrow 2}^{\mathrm{F}}, \mathrm{T}_{1 \leftrightarrow 3}^{\mathrm{F}}\right\}\end{array}$ & $\begin{array}{c}\left\{\mathbf{p}_{L 1}, \mathbf{p}_{L 2}, \mathbf{p}_{L 3}, \mathbf{p}_{L 4}\right\} \\
\left\{\mathrm{T}_{1 \leftrightarrow 2}^{\mathrm{F}}, \mathrm{T}_{1 \leftrightarrow 3}^{\mathrm{F}}, \mathrm{T}_{4 \rightarrow 1}^{\mathrm{F}}\right\}\end{array}$ \\
\hline Vehicle 2: $L F R_{r e f_{-}}^{d}$ & $\begin{array}{l}L F G_{2} \\
L F T_{2} \\
\end{array}$ & $\begin{array}{c}\left\{\mathbf{p}_{L 1}, \mathbf{p}_{L 2}, \mathbf{p}_{L 3}, \mathbf{p}_{L 4}\right\} \\
\left\{\mathrm{T}_{1 \leftrightarrow 2}^{\mathrm{F}}, \mathrm{T}_{2 \leftrightarrow 3}^{\mathrm{F}}, \mathrm{T}_{4 \rightarrow 2}^{\mathrm{F}}\right\} \\
\end{array}$ & $\begin{array}{c}\left\{\mathbf{p}_{L 1}, \mathbf{p}_{L 2}, \mathbf{p}_{L 3}, \mathbf{p}_{L 4}\right\} \\
\left\{\mathrm{T}_{1 \leftrightarrow 2}^{\mathrm{F}}, \mathrm{T}_{2 \leftrightarrow 3}^{\mathrm{F}}, \mathrm{T}_{4 \rightarrow 2}^{\mathrm{F}}\right\}\end{array}$ \\
\hline Vehicle 3: $L F R_{r e f_{-}}^{d}$ & $\begin{array}{l}L F G_{3} \\
L F T_{3} \\
\end{array}$ & $\begin{array}{c}\left\{\mathbf{p}_{L 1}, \mathbf{p}_{L 2}, \mathbf{p}_{L 3}\right\} \\
\left\{\mathrm{T}_{2 \leftrightarrow 3}^{\mathrm{F}}, \mathrm{T}_{1 \leftrightarrow 3}^{\mathrm{F}}\right\} \\
\end{array}$ & $\begin{array}{l}\left\{\mathbf{p}_{L 1}, \mathbf{p}_{L 2}, \mathbf{p}_{L 3}\right\} \\
\left\{\mathrm{T}_{2 \leftrightarrow 3}^{\mathrm{F}}, \mathrm{T}_{1 \leftrightarrow 3}^{\mathrm{F}}\right\} \\
\end{array}$ \\
\hline \multicolumn{2}{|c|}{ Formation vehicles } & Fig. $6(\mathrm{C})$ & Fig. 6 (D) \\
\hline Vehicle 1: $L F R_{r e f_{-} 1}^{d}$ & $\begin{array}{l}L F G_{1} \\
L F T_{1} \\
\end{array}$ & $\begin{array}{r}\left\{\mathbf{p}_{L 1}, \mathbf{p}_{L 2}, \mathbf{p}_{L 3}, \mathbf{p}_{L 4}\right\} \\
\left\{\mathrm{T}_{1 \leftrightarrow 2}^{\mathrm{F}}, \mathrm{T}_{1 \leftrightarrow 3}^{\mathrm{F}}, \mathrm{T}_{4 \rightarrow 1}^{\mathrm{F}}\right\}\end{array}$ & $\begin{array}{l}\left\{\mathbf{p}_{L 1}, \mathbf{p}_{L 2}, \mathbf{p}_{L 3}\right\} \\
\left\{\mathrm{T}_{1 \leftrightarrow 2}^{\mathrm{F}}, \mathrm{T}_{1 \leftrightarrow 3}^{\mathrm{F}}\right\}\end{array}$ \\
\hline Vehicle 2: $L F R_{r e f_{-} 2}^{d}$ & $\begin{array}{l}L F G_{2} \\
L F T_{2}\end{array}$ & $\begin{array}{l}\left\{\mathbf{p}_{L 1}, \mathbf{p}_{L 2}, \mathbf{p}_{L 3}\right\} \\
\left\{\mathrm{T}_{1 \leftrightarrow 2}^{\mathrm{F}}, \mathrm{T}_{2 \leftrightarrow 3}^{\mathrm{F}}\right\}\end{array}$ & $\begin{array}{l}\left\{\mathbf{p}_{L 1}, \mathbf{p}_{L 2}, \mathbf{p}_{L 3}\right\} \\
\left\{\mathrm{T}_{1 \leftrightarrow 2}^{\mathrm{F}}, \mathrm{T}_{2 \leftrightarrow 3}^{\mathrm{F}}\right\}\end{array}$ \\
\hline Vehicle 3: $L F R_{r e f_{-} 3}^{d}$ & $\begin{array}{l}L F G_{3} \\
L F T_{3}\end{array}$ & $\begin{array}{l}\left\{\mathbf{p}_{L 1}, \mathbf{p}_{L 2}, \mathbf{p}_{L 3}\right\} \\
\left\{\mathrm{T}_{2 \leftrightarrow 3}^{\mathrm{F}}, \mathrm{T}_{1 \leftrightarrow 3}^{\mathrm{F}}\right\}\end{array}$ & $\begin{array}{l}\left\{\mathbf{p}_{L 1}, \mathbf{p}_{L 2}, \mathbf{p}_{L 3}\right\} \\
\left\{\mathrm{T}_{2 \leftrightarrow 3}^{\mathrm{F}}, \mathrm{T}_{1 \leftrightarrow 3}^{\mathrm{F}}\right\}\end{array}$ \\
\hline
\end{tabular}

If the original formation system including vehicles 1,2 and 3 is stable and each vehicle can efficiently track the local reference formation $L F R_{r e f_{-} i}^{d}$, the external collision problem can then be solved. Similar to Algorithm 1, the related algorithm for the $i^{t h}$ vehicle is expressed as Algorithm 2, which is executed by each vehicle to avoid any UOs, and consequently their joint efforts implement the external collision avoidance. Finally, once the UO is avoided, the formation system will restore to its original task.

\section{Simulation STUdies}

The formation-based collision avoidance approach is discussed above in terms of solving the intragroup and external collision problems. It was shown that when a vehicle's alert area is invaded by any other vehicles or UOs, the current formation representation could be temporarily replaced by a sequence of intermediate representations until the UO leaves away. In order to implement this approach, two aspects of measures are needed to be adopted. The first one is to select appropriate intermediate formations, whilst the other is to maintain the stability of the multi-vehicle formation system during the transformation process. This implies that the collision avoidance problem is translated into the formation stability problem. In this section, the extension-decomposition-aggregation (EDA) scheme proposed in [26] is employed to design the decentralised multi-vehicle formation controllers for stabilising the corresponding formation and subsequently supporting the formation-based collision avoidance. The associated simulation studies are discussed in terms of the intra-group and external collision avoidance for multi-UAV formation manoeuvring.

\section{A. Implementation of decentralised formation controller}

For simplicity, it is assumed that the dynamic model of the marine robot or vehicle is $\ddot{p}=u$, where $p$ denotes the position of the marine vehicle, and $u$ is the acceleration. For the EDA formation control method [26], the so-called model of the employed individual augmented subsystem (IAS) is given as $\ddot{\theta}=k_{1} \theta+k_{2} u+k_{3} w$, where $\theta$ is a specifically designed variable to indirectly reflect formation changes, $u$ is the acceleration of the marine vessel, and $w$ denotes the dynamics of the local-formation error. The complete IAS model can then be constructed by combining the above equations, and is given by (3),

$$
\left\{\begin{array}{l}
\ddot{p}=u \\
\ddot{\theta}=k_{1} \theta+k_{2} u+k_{3} w
\end{array}\right.
$$


Input: Alert area radius $r_{V i}$ of the $i^{\text {th }}$ vehicle, current reference $L F R_{\text {ref_i }}$, current real $L F R_{\text {real }} i$. Note that $L F R$ contains of the related local formation geometry $(L F G)$ and formation topology $(L F T)$.

Output: Next step reference local formation representation $L F R_{r e f_{-} i}$

1 Calculate the current position of the $i^{t h}$ vehicle $\mathbf{p}_{L i}$ based on $L F R_{\text {real }} i$;

2 Calculate the current position of the $j^{t h}$ vehicle $\mathbf{p}_{L j}^{r}$ based on $L F R_{\text {real }} i$;

3 Calculate the reference position of the $j^{t h}$ vehicle $\mathbf{p}_{L j}^{d}$ based on $L F R_{\text {ref }}{ }_{-}$;

4 Determine the relative distance: $\Delta d_{\text {real }}=\left\|\mathbf{p}_{L i} \mathbf{p}_{L j}^{r}\right\|$;

5 if $\Delta d_{\text {real }} \leq r_{V i}$ then

6 Achieve the crossing point coordinate $\mathbf{p}_{\text {cross }}$ between the alert area circle and the extended line of the vector $\overrightarrow{\mathbf{p}_{L i} \mathbf{p}_{L j}^{r}}$;

$7 \quad$ Based on $L F R_{\text {real }} i$, determine whether there exists a direct reaction to the $j^{\text {th }}$ vehicle;

$8 \quad$ Add a reaction topology of the $i^{\text {th }}$ vehicle relative to the $j^{\text {th }}$ vehicle;

$9 \quad$ Update the reference local formation topology: $L F T_{\text {ref } i}$;

10 Update the desired position of the $j^{t h}$ vehicle: $\mathbf{p}_{L j}^{d}=\mathbf{p}_{\text {cross }}$;

11 Update the reference local formation geometry: $L F G_{r e f_{-} i}$;

12 Update the reference local formation representation: $L F R_{r e f_{-} i}=L F R_{r e f_{-} i}^{d}$;

13 return the updated $L F R_{\text {ref_i }}$;

14 else

15 | return original reference local formation representation: $L F R_{r e f_{-} i}$;

16 end

Algorithm 2: Algorithm of external collision avoidance for the $i^{\text {th }}$ vehicle

The state vector of each IAS is defined as $\mathbf{x}=[p, \dot{p}, \theta, \dot{\theta}]^{T}$, and (3) can be rewritten as (4).

$$
\frac{d}{d t}\left[\begin{array}{c}
p \\
\dot{p} \\
\theta \\
\dot{\theta}
\end{array}\right]=\underbrace{\left[\begin{array}{cccc}
0 & 1 & 0 & 0 \\
0 & 0 & 0 & 0 \\
0 & 0 & 0 & 1 \\
0 & 0 & k_{1} & 0
\end{array}\right]}_{A}\left[\begin{array}{c}
p \\
\dot{p} \\
\theta \\
\dot{\theta}
\end{array}\right]+\underbrace{\left[\begin{array}{c}
0 \\
1 \\
0 \\
k_{2}
\end{array}\right]}_{B_{2}} u+\underbrace{\left[\begin{array}{c}
0 \\
0 \\
0 \\
k_{3}
\end{array}\right]}_{B_{1}} w
$$

Based on the achieved formation stability result [26], the controllers for maintaining the stability of the IASs should be designed to stabilise the whole formation which may include the UOs. Here, the exogenous input $w$ is derived from the formation change or error, and is considered as a bounded disturbance which needs to be rejected by the formation control law for stabilising desired formation. The output feedback $H_{\infty}$ controller $\left[A_{k}, B_{k}, C_{k}, D_{k}\right]$ in (5) is then designed to stabilise the relevant IASs under the bounded disturbance condition [27]. The purpose is to minimise the exogenous impact, i.e. to maximise the robust stability and performance of the IAS.

$$
\begin{aligned}
& \dot{\hat{x}}=A_{k} \hat{x}+B_{k} y \\
& u=C_{k} \hat{x}+D_{k} y
\end{aligned}
$$

Here, $\hat{x}$ denotes the states of controller, which feeds the measurements $y$ back to the control signal $u$ of the plant so that the closed-loop controlled system is internally stable and satisfies the desired performance specifications.

Note that the above decentralised $H_{\infty}$ controller is sufficient to maintain the formation stability, but it cannot necessarily guarantee zero steady-state formation error, because of system type of the transfer function from the input $u$ to the target variable $\theta$. Since the variation of $\theta$ is a reflection of the formation 
changing, the problem can be reduced into a standard regulation of the variable $\theta$. The PI-type compensator $D(s)=K_{p}\left(1+K_{i} / s\right)$ is thus employed for eliminating the steady-state formation error.

The parameter values of the IAS system are heuristically chosen as: $k_{1}=24.5, k_{2}=-2.5, k_{3}=8.3$, but can also be related to the dynamics of the marine robot to be controlled. The matrices of the model in (4) are then given by (6).

$$
A=\left[\begin{array}{cccc}
0 & 1 & 0 & 0 \\
0 & 0 & 0 & 0 \\
0 & 0 & 0 & 1 \\
0 & 0 & 24.525 & 0
\end{array}\right], B_{1}=\left[\begin{array}{c}
0 \\
0 \\
0 \\
8.33
\end{array}\right], B_{2}=\left[\begin{array}{c}
0 \\
1 \\
0 \\
-2.5
\end{array}\right]
$$

The Robust Control Toolbox in Matlab can be conveniently used to calculate the relevant output feedback controller matrices. The achieved controller matrices are given in (7).

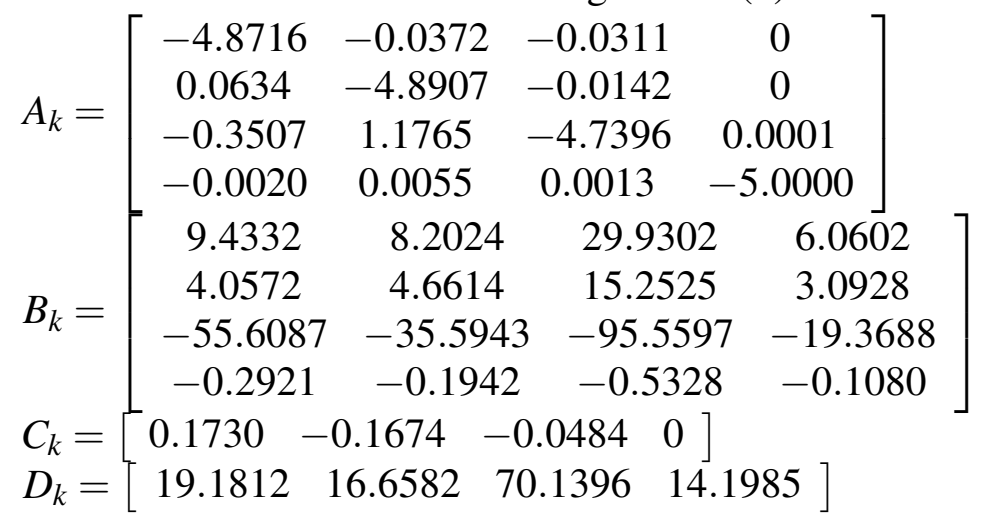

The parameters of the PI compensator were heuristically chosen as $K_{p}=10, K_{i}=15$. The above controller matrices and parameter values were applied to the following simulation.

\section{B. Formation manoeuvre and collision avoidance}

In dealing with a formation containing multiple vehicles there always exists the possibility of collision between vehicles within the formation and between vehicles and external UOs. In this section, the formation-based collision avoidance approach presented in Section III will be implemented in a multirobot formation to evaluate its feasibility and integral performance. In addition, the relationship between formation stability and the implementation of the collision avoidance will also be analysed.

Based on the known obstacles map, the DPSS path planning approach [28] was first employed to calculate the waypoints and subsequently a feasible path from start position to goal position. The multirobot formation was then tasked to navigate all the waypoints provided in Table $\mathrm{V}$, as well as avoiding collisions with any UOs.

TABLE V

WAYPOINTS OF FORMATION MANOEUVRE TRACKING

\begin{tabular}{ccccccc}
\hline$x(m)$ & 6 & 22 & 45 & 81 & 81 & 56 \\
\hline$y(m)$ & 6 & 45 & 80 & 80 & 24 & 6 \\
\hline
\end{tabular}

Some obstacles were also assumed to be present along the path and hence formation changes were required to avoid them. Assuming that the obstacle positions were known a priori, formation changes were obtained off-line and are shown in Fig. 7, where robot 2 is the reference vehicle (RV) [29] and robots 1 and 3 are its designated neighbours. Specifically, Fig. 7 (A) denotes the reference formation representation, and Fig. 7 (B) and Fig. 7 (C) for the intermediate formation representations. Note that the intermediate formation geometry is only used for avoiding obstacles.

The given map, obstacles and the trajectory of the group of vehicles are displayed in Fig 8 showing successful formation maintenance and formation changes whilst avoiding collisions with obstacles. During 


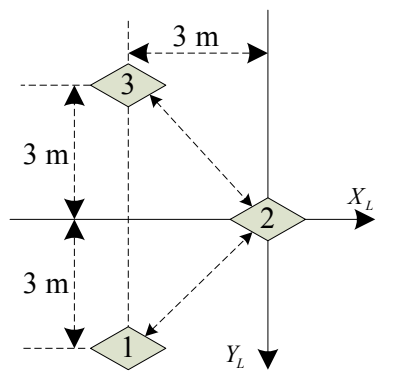

(A) Reference formation representation

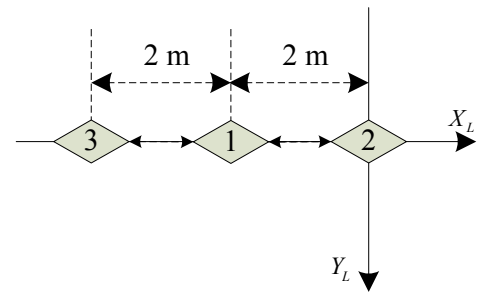

(B) Intermediate formation representation

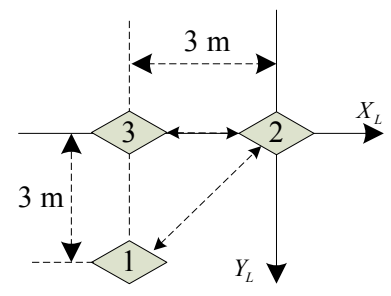

(C) Intermediate formation representation

Fig. 7. Formation representation during multi-robot manoeuvring

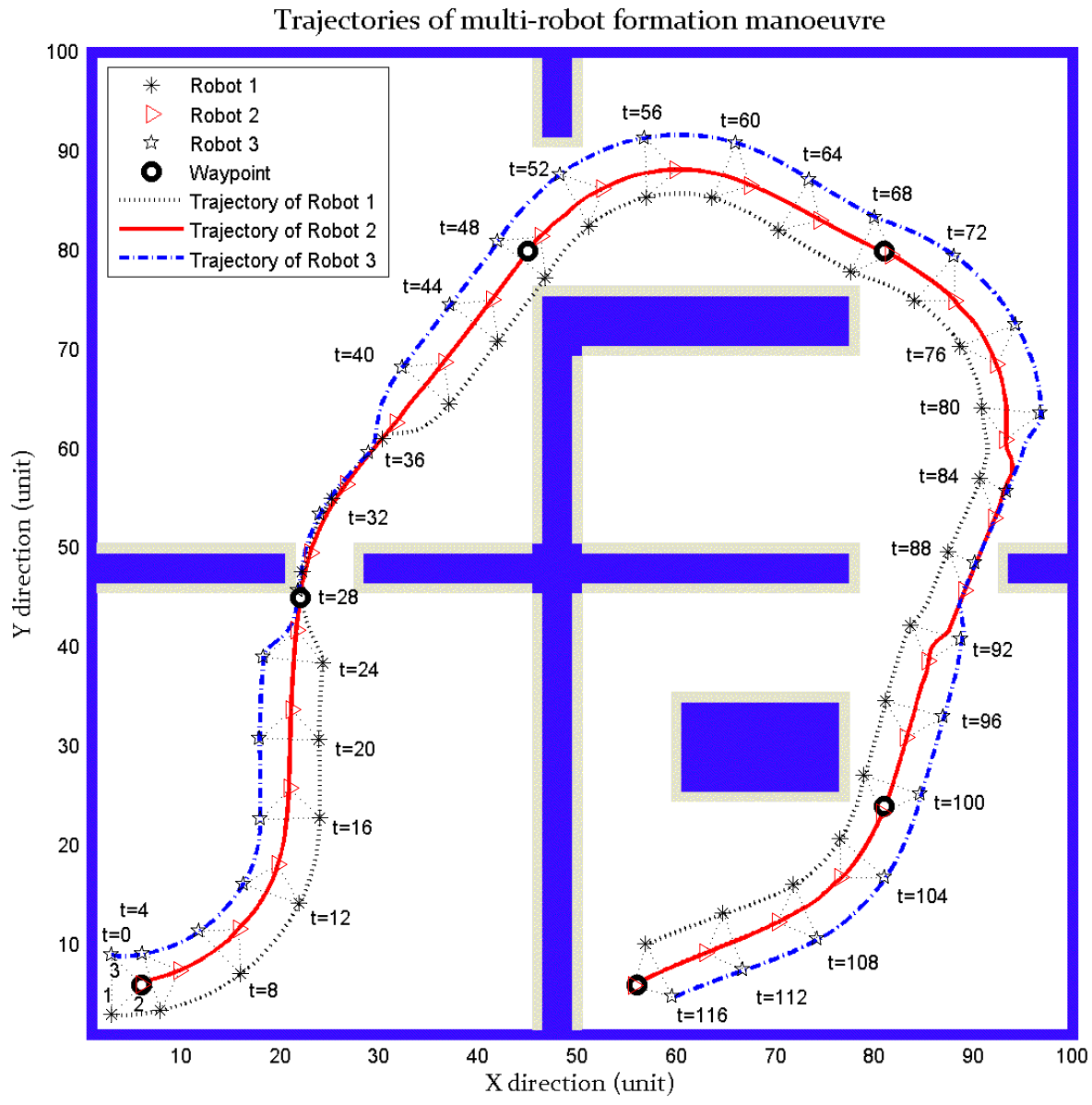

Fig. 8. Trajectories of multi-robot formation manoeuvre

the time intervals $t=24 s \sim 36 s$ and $t=80 s \sim 92 s$, the formation geometry shown in Fig. 7 (B) and (C) were respectively adopted to negotiate forward narrow passages. After successfully navigating the passages, the group of robots restore to the original formation as shown in Fig. 7 (A).

In addition, the speed and heading dynamics of all the vessels in the formation are shown in Fig. 9, whereas the formation errors for robot 1 and robot 3 relative to robot 2 are displayed in Fig. 10. The following observations could be made:

1) The formation with 3 marine robots remained stable during all turning manoeuvres and successfully 
tracked the given waypoints in addition to switching the formations. Hence the proposed formationbased collision avoidance strategy can be used to deal with intra-group as well as and external collision problems.

2) Velocity variation with the formation turning or changing could be observed as it was required to maintain the positions of the robots within the new desired formation as quickly as possible. All craft approached the reference speed of 4 knots once the new formation shape was achieved.

3) During the collision avoidance phase between vehicles or between vehicles and obstacles, the proposed evasive algorithm drives the two vehicles away from each other, and prevents entry into their respective danger zones.

4) The individual formation errors depicted in Fig. 10 eventually converged to zero after achieving the desired formation.
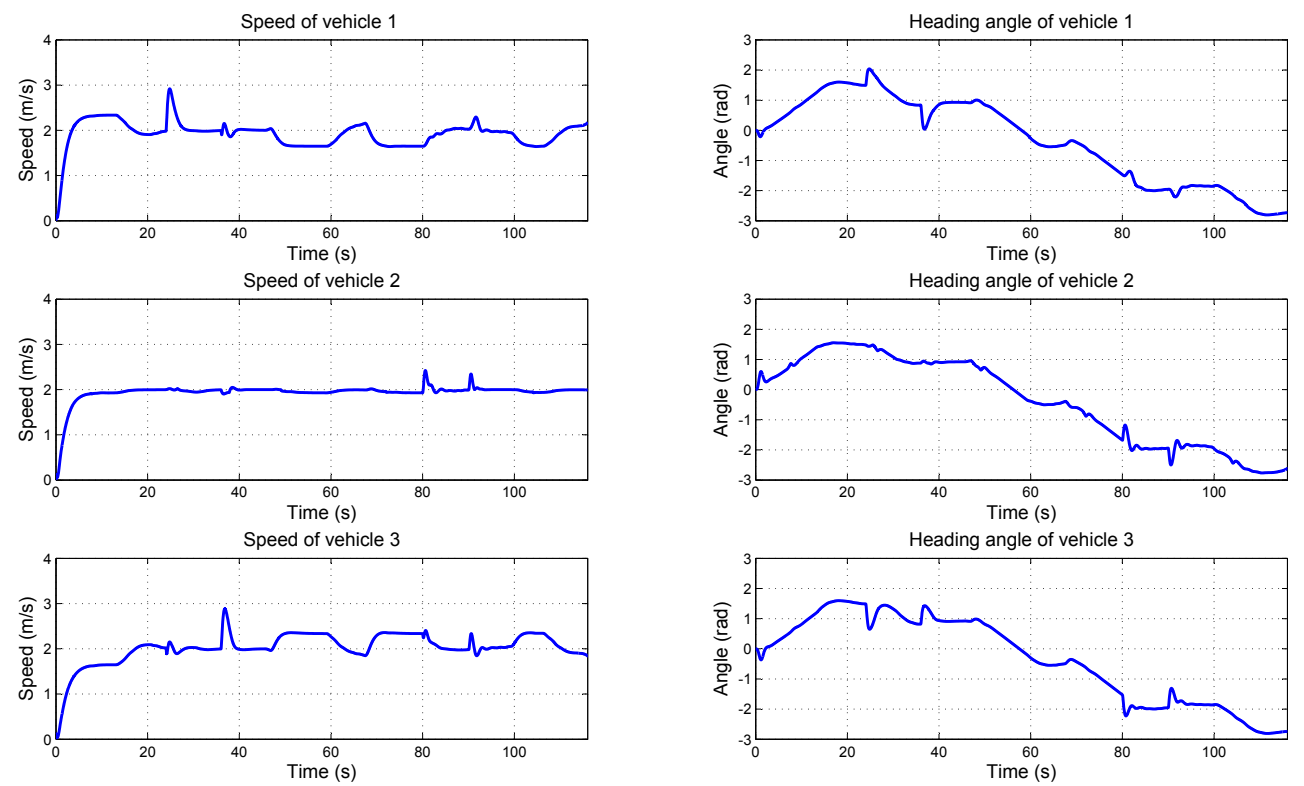

Fig. 9. Variation in speeds and orientations
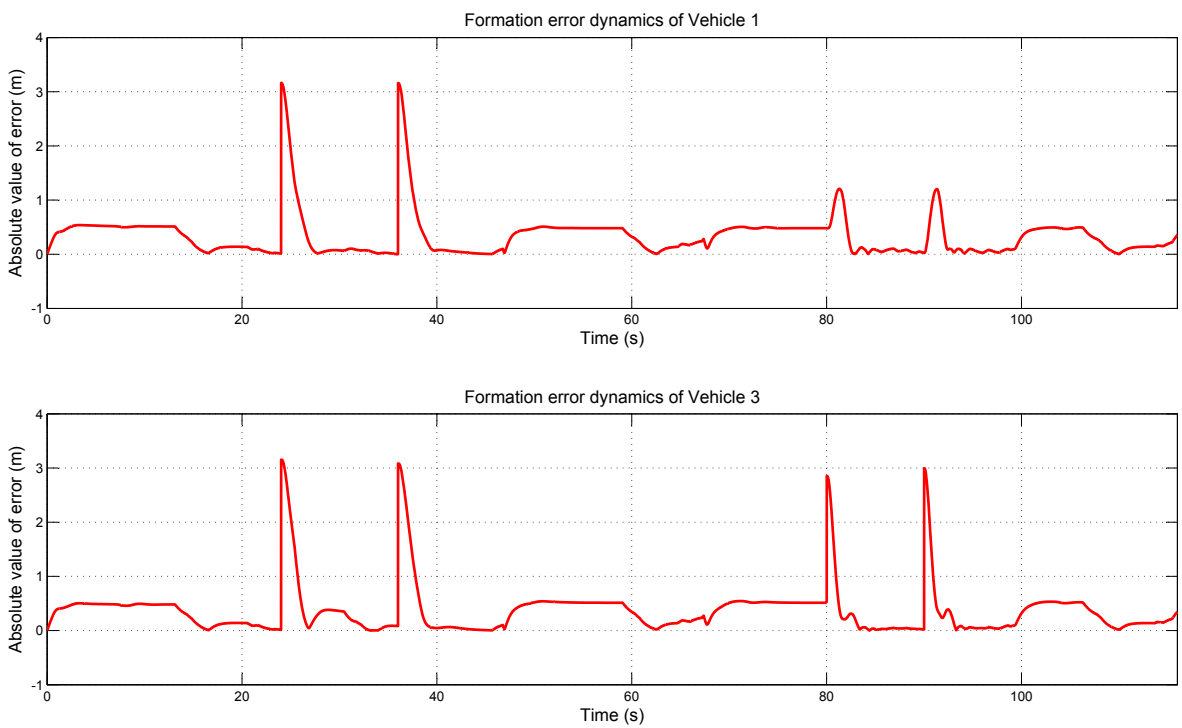

Fig. 10. Formation error of robot 1 and robot 3 
In summary, the proposed formation-based collision avoidance strategy can be conveniently employed to solve the intra-group and external collision problems. The multi-robot formation controller supports not only the formation manoeuvres, but also the implementation of the formation-based collision avoidance. It can be concluded that having satisfied the stability of the augemented formation (by the addition of UOs), the problem of collision avoidance can be translated into the formation control problem.

\section{CONCLUding REMARKS}

This paper focused on the collision avoidance problem for a group of cooperating vehicles. It is demonstrated that the traditional formation-based intra-group and external collision avoidance problem, normally solved separately, is translated into a multi-vehicle formation stability problem. The EDA formation control strategy is then adopted to stabilise the corresponding original and new expanded formations. Simulations are performed to verify the feasibility and effectiveness of the intra-group and external collision avoidance strategy, and indicate the flexibility and wider applicability in dealing with the general collision avoidance problem. Future works include the self-construction protocol, formation topology criteria for collision avoidance, and further application of the formation-based collision avoidance approach to other vehicles, i.e. collision avoidance of UAVs formation flight in three dimensions, and solve the collision avoidance problem in 3-D.

\section{ACKNOWLEDGMENT}

This work was supported by the EPSRC under UK-China Science Bridge Grant (EP/G042594/1), Shanghai Municipal Commission Of Economy and Informatization under Shanghai Industry-UniversityResearch Collaboration Grant (CXY-2013-71), the Science and Technology Commission of Shanghai Municipality under "Yangfan Program" (14YF1408600), and the National Natural Science Foundation of China (Grant No. 61403244).

\section{REFERENCES}

[1] "RMS titanic maritime disaster," last accessed: 09/04/2014.

[2] "Überlingen mid-air collision," last accessed: 09/04/2014.

[3] A. Tsourdos, B. White, and M. Shanmugavel, Cooperative path planning of unmanned aerial vehicles. John Wiley \& Sons, 2011.

[4] J. Hebert, D. Jacques, M. Novy, and M. Pachter, "Cooperative control of UAVs," in Proc. the 2001 AIAA Guidance, Navigation and Control Conference, Montreal, Canada, 2001.

[5] M. Zabarankin, S. Uryasev, and P. Pardalos, Optimal risk path algorithms in cooperative control and optimization. Kluwer Academic, 2002.

[6] A. R. Washburn, "Continuous autorouters with an application to submarines," in Research report, NPSOR-91-05, Naval Postgraduate School, Monterey, USA, 1990.

[7] A. D. Zeitlin and M. P. McLaughlin, "Safety of cooperative collision avoidance for unmanned aircraft," IEEE Aerospace and Electronics Magazine, pp. 9-13, 2007.

[8] J. Kuchar and L. Yang, "A review of conflict detection and resolution modeling methods," IEEE Transactions on Intelligent Transportation Systems, vol. 1, no. 4, pp. 179-189, 2000.

[9] H. I. Yang and Y. J. Zhao, "Trajectory planning for autonomous aerospace vehicles amid obstacles and conflicts," Journal of Guidance, Control and Dynamics, vol. 27, pp. 997-1008, 2004.

[10] F. Lamiraux, D. Bonnafous, and O. Lefebvre, "Reactive path deformation for nonholonomic mobile robots," IEEE Transactions on Robotics, vol. 20, no. 6, pp. 967-977, 2004.

[11] M. Shanmugavel, A. Tsourdos, R. Żbikowski, and B. White, "Path planning of multiple UAVs in an environment of restricted regions," in Proc. ASME Int. Mechanical Engineering Congress and Exposition (IMECE2005), Orlando, USA, 2005.

[12] L. Pallottino, V. G. Scordio, A. Bicchi, and E. Frazzoli, "Decentralized cooperative policy for conflict resolution in multivehicle systems," IEEE Transactions on Robotics, vol. 23, no. 6, pp. 1170-1183, 2007.

[13] A. Pongpunwattana and R. Rysdyk, "Real-time planning for multiple autonomous vehicles in dynamic uncertain environments," Journal of Aerospace Computing, Information, and Communication, vol. 1, no. 12, pp. 580-604, 2004.

[14] S. J. Guy, J. Chhugani, C. Kim, N. Satish, M. Lin, D. Manocha, and P. Dubey, "Highly parallel collision avoidance for multi-agent simulation," in Proc. ACM SIGGRAPH Euro-graphics symposium on computer animation, September 3-5 2009, pp. 345-350.

[15] J. O. Kim and P. K. Khosla, "Real-time obstacle avoidance using harmonic potential functions," IEEE Transactions on Robotics and Automation, vol. 8, pp. 338-349, 1992.

[16] S. Wang and H. Schaub, "Spacecraft collision avoidance using coulomb forces with separation distance and rate feedback," Journal of Aerospace Computing, Information, and Communication, vol. 31, pp. 740-750, 2008. 
[17] S. Mastellone, D. M. Stipanovic, C. R. Graunke, K. A. Intlekofer, and M. W. Spong, "Formation control and collision avoidance for multi-agent non-holonomic systems: Theory and experiments," The International Journal of Robotics Research, vol. 27, no. 1, pp. 107-126, 2008

[18] E. Lalish and K. A. Morgansen, "Distributed reactive collision avoidance," Autonomous Robots, vol. 32, pp. 207-226, 2012.

[19] H. Kowshik, D. Caveney, and P. R. Kumar, "Provable systemwide safety in intelligent intersections," IEEE Transactions on vehicular technology, vol. 60, no. 3, pp. 804-818, 2011.

[20] M. Braännstroöm, E. Coelingh, and J. Sjoöberg, "Model-based threat assessment for avoiding arbitrary vehicle collisions," IEEE Transactions on intelligent transportation systems, vol. 11, no. 3, pp. 658-669, 2010.

[21] D. Greene, J. Liu, J. Reich, Y. Hirokawa, A. Shinagawa, H. Ito, and T. Mikami, "An efficient computational architecture for a collision early warning system for vehicles, pedestrians, and bicyclists," IEEE Transactions on intelligent transportation systems, vol. 12, no. 4, pp. 942-953, 2011.

[22] G. Fasano, D. Accardo, and A. Moccia, "Multi-sensor-based fully autonomous non-cooperative collision avoidance system for unmanned air vehicles," Journal of Aerospace Computing, Information, and Communication, vol. 5, no. 10, pp. 338-360, 2008.

[23] Y. Eun and H. Bang, "Cooperative control of multiple unmanned aerial vehicles using the potential field theory," Journal of Aircraft, vol. 43, no. 6, pp. 1805-1814, 2006.

[24] A. Fujimori, Y. Ogawa, and P. N. Nikiforuk, "A modification of cooperative collision avoidance for multiple robots using the avoidance circle," Proc. of the Institution of Mechanical Engineers, Part I: Journal of Systems and Control Engineering, vol. 216, no. 3, pp. 291-299, 2002.

[25] R. M. Murray, "Recent research in cooperative control of multi-vehicle systems," Journal of Dynamic Systems, Measurement, and Control, vol. 129, no. 5, pp. 571-583, 2007.

[26] A. Yang, W. Naeem, G. W. Irwin, and K. Li, "Stability analysis and implementation of a decentralised formation control strategy for unmanned vehicles," IEEE Transactions on Control Systems Technology, vol. 22, no. 2, pp. 706-720, 2014.

[27] K. Zhou, J. Doyle, and K. Glover, Robust and optimal control. Prentice Hall, 1996.

[28] A. Yang, Q. Niu, W. Zhao, K. Li, and G. W. Irwin, "An efficient algorithm for grid-based robotic path planning based on priority sorting of direction vectors," in Proc. international conference on life system modelling and simulation, LSMS 2010, Wuxi, China, 2010, pp. 456-466.

[29] A. Yang, W. Naeem, G. W. Irwin, and K. Li, "A decentralised control strategy for formation flight of unmanned aerial vehicles," in Proc. 2012 UKACC International Conference on Control, Cardiff, UK, September 3-5 2012, pp. 345-350. 